International Electronic Journal of Algebra

VOLUMe 21 (2017) 137-163

\title{
ON SOME PROPERTIES OF CHEBYSHEV POLYNOMIALS AND THEIR APPLICATIONS
}

\author{
Jun $\mathrm{Hu}$ and Yabo $\mathrm{Wu}$ \\ Received: 10 June 2016; Revised: 20 October 2016 \\ Communicated by Abdullah Harmanc1
}

\begin{abstract}
In this paper we investigate certain normalized versions $S_{k, F}(x)$, $\widetilde{S}_{k, F}(x)$ of Chebyshev polynomials of the second kind and the fourth kind over a field $F$ of positive characteristic. Under the assumption that (char $F, 2 m+$ $1)=1$, we show that $\widetilde{S}_{m, F}(x)$ has no multiple roots in any one of its splitting fields. The same is true if we replace $2 m+1$ by $2 m$ and $\widetilde{S}_{m, F}(x)$ by $S_{m-1, F}(x)$. As an application, for any commutative ring $R$ which is a $\mathbb{Z}\left[1 / n, 2 \cos (2 \pi / n), u^{ \pm 1 / 2}\right]$-algebra, we construct an explicit cellular basis for the Hecke algebra associated to the dihedral groups $I_{2}(n)$ of order $2 n$ and defined over $R$ by using linear combinations of some Kazhdan-Lusztig bases with coefficients given by certain evaluations of $\widetilde{S}_{k, R}(x)$ or $S_{k, R}(x)$.
\end{abstract}

Mathematics Subject Classification (2010): 20C08, 12D10

Keywords: Chebyshev polynomials, dihedral group, Hecke algebras, cellular basis

\section{Introduction}

The Chebyshev polynomials are a sequence of important orthogonal polynomials over $\mathbb{Z}$ which are related to de Moivre's formula and which can be defined recursively. They have found many important applications in diverse areas of mathematics such as ordinary and partial differential equations, analysis and approximation theory. In past three decades these polynomials also come up in several places in nearby areas of representation theory. For example, they appear in the criterion for semisimplicity of Temperley-Lieb and Jones algebras [4], [5], in giving the dimension of a centralizer algebra of a Temperley-Lieb algebra, and in calculating the decomposition of a Brauer algebra module into Temperley-Lieb algebra modules [2], and in constructing irreducible representations of the semisimple Hecke algebra associated to the dihedral groups [3]. However, it seems to us that all of these applications only use property of Chebyshev polynomials over the complex

This work was carried out under the support of the National Natural Science Foundation of China (NSFC 11525102). 
numbers field (or any field of characteristic 0). To the best of our knowledge, the property of Chebyshev polynomials over field of positive characteristic are not wellstudied and exploited in the literatures before. In this paper we shall study some of their properties for the normalized versions $S_{n}(x):=U_{n}(x / 2), \widetilde{S}_{n}(x):=W_{n}(x / 2)$ of Chebyshev polynomials of the second kind and of the fourth kind over certain fields of positive characteristic, where $U_{n}(x)$ and $W_{n}(x)$ are the Chebyshev polynomials of the second kind and of the fourth kind respectively. As an application, we shall construct an explicit cellular basis for the Hecke algebra $H_{q, R}\left(W_{n}\right)$ associated to the dihedral group $W_{n}:=I_{2}(n)$ of order $2 n$ over any commutative ring $R$ which is a $\mathbb{Z}\left[\frac{1}{n}, 2 \cos \left(\frac{2 \pi}{n}\right), u^{ \pm 1 / 2}\right]$-algebra.

The content is organised as follows. In Section 2 we investigate the Chebyshev polynomials over fields of positive characteristic. We show (in Lemmas 2.8 and 2.9) that under the assumption that $(\operatorname{char} F, 2 m+1)=1($ respectively, $(\operatorname{char} F, 2 m)=$ 1 ), the normalised Chebyshev polynomial $\widetilde{S}_{m, F}(x)$ of the fourth kind (respectively, $S_{m-1, F}(x)$ of the second kind) has no multiple roots over any one of its splitting fields. As a result, we show that for any $n \in \mathbb{N}$ and $1 \leq j<l \leq[(n-1) / 2]$, $2 \cos (2 l \pi / n)-2 \cos (2 j \pi / n)$ is invertible in $\mathbb{Z}\left[\frac{1}{n}, 2 \cos \left(\frac{2 \pi}{n}\right)\right]$. In Section 3, we apply these results to construct a cellular basis of the Hecke algebra associated to the dihedral group $W_{n}:=I_{2}(n)$ of order $2 n$. We show (in Lemmas 2.10 and 2.11) that for any commutative ring $R$ which is a $\mathbb{Z}\left[\frac{1}{n}, 2 \cos \left(\frac{2 \pi}{n}\right), u^{ \pm 1 / 2}\right]$-algebra, certain linear combinations of some Kazhdan-Lusztig bases can form a cellular basis of the Hecke algebra $H_{q, R}\left(W_{n}\right)$ over $R$, see Theorems 3.14 and 3.22. The coefficients of each Kazhdan-Lusztig bases are given by a scalar multiple of some evaluation of certain explicit Chebyshev polynomials.

\section{Chebyshev polynomials over fields of positive characteristic}

The purpose of this section is to study certain normalised Chebyshev polynomials over fields of positive characteristic. Let $x$ be an indeterminate over $\mathbb{Z}$.

Definition 2.1. The Chebyshev polynomials $\left\{T_{k}(x)\right\}_{k \geq 0}$ of the first kind are defined recursively by:

$$
T_{0}(x):=1, T_{1}(x):=x, T_{k+1}(x):=2 x T_{k}(x)-T_{k-1}(x), \forall k \geq 1 .
$$

The Chebyshev polynomials $\left\{U_{k}(x)\right\}_{k \geq 0}$ of the second kind are defined recursively by:

$$
U_{0}(x):=1, U_{1}(x):=2 x, U_{k+1}(x):=2 x U_{k}(x)-U_{k-1}(x), \forall k \geq 1 .
$$


The Chebyshev polynomials $\left\{W_{k}(x)\right\}_{k \geq 0}$ of the fourth kind are defined recursively by:

$$
W_{0}(x):=1, W_{1}(x):=2 x+1, W_{k+1}(x):=2 x W_{k}(x)-W_{k-1}(x), \forall k \geq 1 .
$$

Definition 2.2. For each $k \geq 0$, we define

$$
S_{k}(x):=U_{k}(x / 2), \quad \widetilde{S}_{k}(x):=W_{k}(x / 2) .
$$

We shall call $S_{k}(x), \widetilde{S}_{k}(x)$ the normalized Chebyshev polynomials of the second kind and of the fourth kind respectively.

One can check that $S_{0}(x)=1, S_{1}(x)=x$ and

$$
S_{k+1}(x):=x S_{k}(x)-S_{k-1}(x)
$$

while $\widetilde{S}_{0}(x)=1, \widetilde{S}_{1}(x)=x+1$ and

$$
\widetilde{S}_{k+1}(x):=x \widetilde{S}_{k}(x)-\widetilde{S}_{k-1}(x) .
$$

Furthermore, for any $k \geq 0, T_{k}(x), U_{k}(x), S_{k}(x), \widetilde{S}_{k}(x) \in \mathbb{Z}[x] \subset \mathbb{C}[x]$, and

$$
\begin{aligned}
& S_{k}(x)=x^{k}+\text { lower terms, } \quad \widetilde{S}_{k}(x)=x^{k}+\text { lower terms, } \\
& U_{k}(x)=2^{k} x^{k}+\text { lower terms. }
\end{aligned}
$$

Lemma 2.3. $[8,(1.15),(1.18),(2.30 \mathrm{c}),(2.30 \mathrm{~d})]$ For any integer $k \geq 1$, we have that

$$
\widetilde{S}_{k}(x)=W_{k}(x / 2)=U_{k}(x / 2)+U_{k-1}(x / 2)=S_{k}(x)+S_{k-1}(x),
$$

while for any $m \geq 0, W_{m}\left(2 x^{2}-1\right)=U_{2 m}(x)$.

Lemma 2.4. $[8,(2.30 \mathrm{a}),(2.30 \mathrm{c}),(2.30 \mathrm{~d})]$ For any $k \geq 0$, we have that $S_{k}(-2)=$ $U_{k}(-1)=(-1)^{k}(k+1)$, and

$$
\widetilde{S}_{k}(0)=(-1)^{[k / 2]}, U_{2 k}(0)=(-1)^{k}, T_{k}(0)=(-1)^{k} .
$$

Let $R$ be a commutative ring. For any $f(x) \in \mathbb{Z}[x]$, we denote by $f_{R}(x)$ the image of $f(x)$ in $R[x]$ under the natural homomorphism $\mathbb{Z}[x] \rightarrow R[x]$. We set

$T_{k, R}(x):=\left(T_{k}\right)_{R}(x), U_{k, R}(x):=\left(U_{k}\right)_{R}(x), S_{k, R}(x):=\left(S_{k}\right)_{R}(x), \widetilde{S}_{k, R}(x):=\left(\widetilde{S}_{k}\right)_{R}(x)$.

Lemma 2.5. For any integer $k \geq 1$, we have that

$$
\widetilde{S}_{k, R}(x)=S_{k-1, R}(x)+S_{k, R}(x) .
$$

For each $f(x)=\sum_{i=0}^{k} a_{i} x^{i} \in R[x]$, let $f^{\prime}(x):=\sum_{i=1}^{k} i a_{i} x^{i-1} \in R[x]$ be the formal derivative of $f(x)$. 
Lemma 2.6. $[1,8]$ Let $k \in \mathbb{Z}^{\geq 0}$ and $F$ be an arbitrary field. We have that

$$
\begin{aligned}
& T_{k+1, F}^{\prime}(x)=(k+1) U_{k, F}(x), T_{k+2, F}(x)=x T_{k+1, F}(x)-\left(1-x^{2}\right) U_{k, F}(x), \\
& (k+1) T_{k+1, F}(x)-x U_{k, F}(x)=\left(x^{2}-1\right) U_{k, F}^{\prime}(x) .
\end{aligned}
$$

Lemma 2.7. Let $k \in \mathbb{Z}^{\geq 1}$. Then

$$
\begin{aligned}
\left(4-x^{2}\right) \widetilde{S}_{n}^{\prime}(x) & =(n+1) \widetilde{S}_{n-1}(x)-n \widetilde{S}_{n+1}(x)+\widetilde{S}_{n}(x) \\
& =(2 n+1) \widetilde{S}_{n-1}(x)+(1-n x) \widetilde{S}_{n}(x) .
\end{aligned}
$$

In particular, if $F$ is a field with $\operatorname{char} F=2$ and $m \in \mathbb{N}$, then

$$
\begin{aligned}
& -x^{2} \widetilde{S}_{2 m+1, F}^{\prime}(x)=-\widetilde{S}_{2 m+2, F}(x)+\widetilde{S}_{2 m+1, F}(x), \\
& -x^{2} \widetilde{S}_{2 m, F}^{\prime}(x)=\widetilde{S}_{2 m-1, F}(x)+\widetilde{S}_{2 m, F}(x) .
\end{aligned}
$$

Proof. By [8, Chapter 2, Exercise 15],

$$
2\left(1-x^{2}\right) U_{n}^{\prime}(x)=(n+2) U_{n-1}(x)-n U_{n+1}(x) .
$$

Now the lemma follows from the above equality, (2) and the fact that $S_{n}(x)=$ $U_{n}(x / 2)$ and $S_{n}^{\prime}(x)=\frac{1}{2} U_{n}^{\prime}(x / 2)$.

The Chebyshev polynomials over the complex numbers field $\mathbb{C}$ have many nice properties. For example, it is well-known that for any integer $k \geq 1$,

(1) $S_{k}(2 \cos \theta)=\frac{\sin (k+1) \theta}{\sin \theta}$;

(2) the roots of the polynomial $S_{k-1}(x)$ in $\mathbb{C}$ are

$$
2 \cos \frac{j \pi}{k}, \quad j=1,2, \cdots, k-1 ;
$$

(3) the roots of the polynomial $\widetilde{S}_{k}(x):=S_{k}(x)+S_{k-1}(x)$ in $\mathbb{C}$ are

$$
2 \cos \frac{2 j \pi}{2 k+1}, \quad j=1,2, \cdots, k .
$$

The following lemma gives a positive characteristic analogue of the property c) for the Chebyshev polynomials over fields of positive characteristic.

Lemma 2.8. Let $F$ be an arbitrary field and $k \in \mathbb{Z}^{\geq 0}$ such that either char $F=0$ or char $F$ is coprime to $2 k+1$. Then the polynomial $\widetilde{S}_{k, F}(x)$ over $F$ has $k$-distinct roots in any one of its splitting field.

Proof. If $k=0$, then $\widetilde{S}_{k, F}(x)=x+1$ has a unique root -1 . So there is nothing to prove. Henceforth we assume that $k \geq 1$.

We first assume that char $F \neq 2$. Then $2 \cdot 1_{F}$ is invertible in $F$. By Lemma 2.3, $\widetilde{S}_{k, F}(x)=W_{k, F}(x / 2)$. To prove the lemma, it suffices to show that $W_{k, F}(x)$ over $F$ has $k$-distinct roots in any one of its splitting field. 
By Lemma 2.3, $W_{k, F}\left(2 x^{2}-1\right)=U_{2 k, F}(x)$ and $W_{k, F}(-1)=U_{2 k, F}(0)= \pm 1$. It follows that $4 x$ is coprime to $U_{2 k, F}(x)=W_{k, F}\left(2 x^{2}-1\right)$ in $F[x]$. Therefore,

$$
\left(W_{k, F}(x), W_{k, F}^{\prime}(x)\right)=1 \text { if and only if }\left(U_{2 k, F}(x), U_{2 k, F}^{\prime}(x)\right)=1 \text {. }
$$

As a result, we see that to prove the lemma, it suffices to show that the $F$-polynomial $U_{2 k, F}(x)$ has $2 k$-distinct roots in any one of its splitting field.

By Lemma 2.6, we have that

$$
(2 k+1) T_{2 k+1, F}(x)-x U_{2 k, F}(x)=\left(x^{2}-1\right) U_{2 k, F}^{\prime}(x) .
$$

By assumption, $(2 k+1) \cdot 1_{F} \neq 0$ in $F$. Therefore, we can deduce that $\left(U_{2 k, F}(x), U_{2 k, F}^{\prime}(x)\right)$ is a factor of $\left(T_{2 k+1, F}(x), U_{2 k, F}(x)\right)$. By Lemma 2.6,

$$
T_{2 k+2, F}(x)=x T_{2 k+1, F}(x)-\left(1-x^{2}\right) U_{2 k, F}(x) .
$$

It follows that $\left(T_{2 k+1, F}(x), U_{2 k, F}(x)\right)$ and hence $\left(U_{2 k, F}(x), U_{2 k, F}^{\prime}(x)\right)$ is a factor of $\left(T_{2 k+2, F}(x), T_{2 k+1, F}(x)\right)$.

On the other hand, by definition, for any $m \geq 1$,

$$
T_{m+1, F}(x):=2 x T_{m, F}(x)-T_{m-1, F}(x) .
$$

It follows that $\left(T_{m+1, F}(x), T_{m, F}(x)\right)$ is always a factor of $\left(T_{m, F}(x), T_{m-1, F}(x)\right)$. Inductively, we can deduce that $\left(T_{m+1, F}(x), T_{m, F}(x)\right)$ is a factor of

$$
\left(T_{1, F}(x), T_{0, F}(x)\right)=(x, 1)=1 .
$$

This proves that $\left(T_{m+1, F}(x), T_{m, F}(x)\right)=1$ for any $m \geq 0$. As a result, we can deduce that $\left(T_{2 k+2, F}(x), T_{2 k+1, F}(x)\right)=1$ and hence $\left(U_{2 k, F}(x), U_{2 k, F}^{\prime}(x)\right)=1$. This implies that $U_{2 k, F}(x)$ has $2 k$-distinct roots in any splitting field of $U_{2 k, F}(x)$, as required.

Now assume that char $F=2$. Suppose that $k=2 m+1$ is an odd integer. Applying Lemma 2.7, we can deduce that $\left(\widetilde{S}_{2 m+1, F}^{\prime}(x), \widetilde{S}_{2 m+1, F}(x)\right)$ is a factor of $\left(\widetilde{S}_{2 m+2, F}(x), \widetilde{S}_{2 m+1, F}(x)\right)$. By (1) and an easy induction on $m$, it is easy to see that

$$
\begin{aligned}
\left(\widetilde{S}_{2 m+2, F}(x), \widetilde{S}_{2 m+1, F}(x)\right) & =\left(\widetilde{S}_{2 m+1, F}(x), \widetilde{S}_{2 m, F}(x)\right)=\cdots=\left(\widetilde{S}_{2, F}(x), \widetilde{S}_{1, F}(x)\right) \\
& =\left(x^{2}+x-1, x+1\right)=1 .
\end{aligned}
$$

In other words, $\left(\widetilde{S}_{2 m+1, F}^{\prime}(x), \widetilde{S}_{2 m+1, F}(x)\right)=1$. So $\widetilde{S}_{2 m+1, F}(x)$ has no multiple roots. If $k=2 m$ is an even integer, the lemma follows from Lemma 2.7 and a similar argument. 
Lemma 2.9. Let $F$ be an arbitrary field and $k \in \mathbb{Z}^{\geq 1}$ such that either $\operatorname{char} F=0$ or char $F$ is coprime to $2 k$. Then the polynomial $S_{k-1, F}(x)$ over $F$ has $(k-1)$-distinct roots in any one of its splitting field.

Proof. If $k=1$ then $S_{k-1, F}(x)=1$ and there is nothing to prove. If $k=2$, then $S_{k-1, F}(x)=x$ has a unique root 0 as required. Henceforth we assume that $k \geq 3$.

By assumption, $2 \cdot 1_{F}$ is invertible in $F$. By definition, $S_{k-1, F}(x)=U_{k-1, F}(x / 2)$. To prove the lemma, it suffices to show that $U_{k-1, F}(x)$ over $F$ has $(k-1)$-distinct roots in any one of its splitting field.

By Lemma 2.6, we have that

$$
k T_{k, F}(x)-x U_{k-1, F}(x)=\left(x^{2}-1\right) U_{k-1, F}^{\prime}(x) .
$$

By assumption, $k \cdot 1_{F} \neq 0$ in $F$. Therefore, we can deduce that $\left(U_{k-1, F}(x), U_{k-1, F}^{\prime}(x)\right)$ is a factor of $\left(T_{k, F}(x), U_{k-1, F}(x)\right)$. By Lemma 2.6,

$$
T_{k+1, F}(x)=x T_{k, F}(x)-\left(1-x^{2}\right) U_{k-1, F}(x) .
$$

It follows that $\left(T_{k, F}(x), U_{k-1, F}(x)\right)$ and hence $\left(U_{k-1, F}(x), U_{k-1, F}^{\prime}(x)\right)$ is a factor of $\left(T_{k+1, F}(x), T_{k, F}(x)\right)$.

On the other hand, by definition, for any $m \geq 1$,

$$
T_{m+1, F}(x):=2 x T_{m, F}(x)-T_{m-1, F}(x) .
$$

It follows that $\left(T_{m+1, F}(x), T_{m, F}(x)\right)$ is always a factor of $\left(T_{m, F}(x), T_{m-1, F}(x)\right)$. Inductively, we can deduce that $\left(T_{m+1, F}(x), T_{m, F}(x)\right)$ is a factor of

$$
\left(T_{1, F}(x), T_{0, F}(x)\right)=(x, 1)=1 .
$$

This proves that $\left(T_{m+1, F}(x), T_{m, F}(x)\right)=1$ for any $m \geq 0$. As a result, we can deduce that $\left(T_{k+1, F}(x), T_{k, F}(x)\right)=1$ and hence $\left(U_{k-1, F}(x), U_{k-1, F}^{\prime}(x)\right)=1$. This implies that $U_{k-1, F}(x)$ has $(k-1)$-distinct roots in any splitting field of $U_{k-1, F}(x)$, as required. This completes the proof of the lemma.

Henceforth, we set

$$
\mathcal{A}:=\mathbb{Z}[1 / n, 2 \cos (2 \pi / n)]
$$

Lemma 2.10. Suppose that $n=2 m+1$. Then for any $1 \leq k<l \leq m$, we have that $2 \cos (2 k \pi / n)-2 \cos (2 l \pi / n)$ is invertible in $\mathcal{A}$. Furthermore,

$$
\prod_{j=1}^{m}(2+2 \cos (2 j \pi / n))=1 .
$$

In particular, for each $1 \leq j \leq m, 2+2 \cos (2 j \pi / 2 m+1)$ is invertible in $\mathcal{A}$. 
Proof. Suppose that $2 \cos (2 k \pi / n)-2 \cos (2 l \pi / n)$ is not invertible in $\mathcal{A}$ for some $1 \leq k<l \leq m$. Let $\mathfrak{m}$ be a maximal ideal of $\mathcal{A}$ which contains $2 \cos (2 k \pi / n)-$ $2 \cos (2 l \pi / n)$. Let $k:=\mathcal{A} / \mathfrak{m}$ be the residue field and $\tau$ be the canonical homomorphism $\mathcal{A} \rightarrow \mathcal{A} / \mathfrak{m}=k$. By construction, we see that $2 m+1$ is invertible in $k$. Applying Lemma 2.8 , we can deduce that $\widetilde{S}_{m, k}(x)$ has no multiple roots. On the other hand, it is clear that

$$
\widetilde{S}_{m, k}(x)=\prod_{j=1}^{m}(x-\tau(2 \cos (2 j \pi / n))) .
$$

It follows that the elements in $\{\tau(2 \cos (2 j \pi / n)) \mid 1 \leq j \leq m\}$ must be pairwise distinct. In particular, $\tau(2 \cos (2 k \pi / n)-2 \cos (2 l \pi / n))=\tau(2 \cos (2 k \pi / n))-\tau(2 \cos (2 l \pi / n))$ is nonzero for any $1 \leq k<l \leq m$, which is a contradiction because $2 \cos (2 k \pi / n)-$ $2 \cos (2 l \pi / n) \in \mathfrak{m}$. This proves that $2 \cos (2 k \pi / n)-2 \cos (2 l \pi / n)$ is invertible in $\mathcal{A}$ for any $1 \leq k<l \leq m$. It remains to prove the second half of the lemma.

For each $1 \leq j \leq m, 2+2 \cos (2 j \pi / n)=(2 \cos (j \pi / n))^{2}$. Since $\cos (j \pi / n)=$ $-\cos ((n-j) \pi / n)$, it follows that

$$
\left(\prod_{j=1}^{m} 2 \cos (j \pi / n)\right)^{2}=\left(\prod_{j=1}^{m} 2 \cos (2 j \pi /(2 m+1))\right)^{2}=1,
$$

where the last equality follows because $\{2 \cos (2 j \pi /(2 m+1)) \mid 1 \leq j \leq m\}$ are all the roots of the monic polynomial $\widetilde{S}_{m}(x)$ and $\widetilde{S}_{m}(0)=(-1)^{[m / 2]}$ by Lemma 2.4 . This completes the proof of the lemma.

Lemma 2.11. Suppose that $n=2 m$. Then for any $1 \leq k<l \leq m-1$, we have that $2 \cos (2 k \pi / n)-2 \cos (2 l \pi / n)$ is invertible in $\mathcal{A}$. Furthermore,

$$
\prod_{j=1}^{m-1}(2+2 \cos (2 j \pi / n))=m
$$

In particular, for each $1 \leq j \leq m-1,2+2 \cos (2 j \pi / 2 m)$ is invertible in $\mathcal{A}$.

Proof. The first half of the lemma can be proved in a similar way as the proof of Lemma 2.10. It remains to prove the second half of the lemma.

Since $\{2 \cos (2 j \pi /(2 m)) \mid 1 \leq j \leq m-1\}$ are all the roots of the monic polynomial $S_{m-1}(x)$, we can deduce that

$$
S_{m-1}(x)=\prod_{j=1}^{m-1}(x-2 \cos (2 j \pi /(2 m))) .
$$

It follows that

$$
\prod_{j=1}^{m-1}(2+2 \cos (2 j \pi / n))=(-1)^{m-1} S_{m-1}(-2)=(-1)^{m-1}(-1)^{m-1} m=m,
$$


as required, where the second last equality follows from Lemma 2.4.

\section{Cellular basis of $H_{q, R}\left(W_{n}\right)$}

In this section we shall use the main result of the last section to construct an explicit cellular basis of the Hecke algebras $H_{q, R}\left(W_{n}\right)$ associated to the dihedral group $W_{n}=I_{2}(n)$ over any commutative ring $R$ which is a $\mathbb{Z}\left[1 / n, 2 \cos (2 \pi / n), u^{ \pm 1 / 2}\right]$ algebra.

We first briefly recall some well-known basic knowledge about the KazhdanLusztig basis and Kazhdan-Lusztig polynomials for the Hecke algebras $H_{u}(W)$ associated to a Coxeter group $W$.

Let $u^{1 / 2}$ be an indeterminate over $\mathbb{Z}$ and $A:=\mathbb{Z}\left[u^{1 / 2}, u^{-1 / 2}\right]$. Let $(W, S)$ be Coxeter system with length function $\ell(?)$ and $H_{u}(W)$ be the associated IwahoriHecke algebra over $A$ with Hecke parameter $u$. By definition, $H_{u}(W)$ is a free $A$ module with an $A$-basis $\left\{T_{w}\right\}_{w \in W}$. The multiplication rule of $H_{u}(W)$ is determined by:

$$
T_{s} T_{w}=\left\{\begin{array}{ll}
T_{s w}, & \text { if } l(s w)>l(w), \\
u T_{s w}+(u-1) T_{w}, & \text { if } l(s w)<l(w) .
\end{array} \quad T_{w} T_{s}=\left(T_{s} T_{w^{-1}}\right)^{*},\right.
$$

where $s \in S, w \in W$, and "*" is the anti-isomorphism of $H_{u}(W)$ which is defined on generators by $T_{w}^{*}:=T_{w^{-1}}$ for any $w \in W$.

Let $a \mapsto \bar{a}$ be the involution of the ring $A$ which is defined by $\overline{u^{1 / 2}}=u^{-1 / 2}$. This extends to an involution $h \mapsto \bar{h}$ of the ring $H_{u}(W)$, defined by

$$
\overline{\sum_{w \in W} a_{w} T_{w}}:=\sum_{w \in W} \overline{a_{w}} T_{w^{-1}}^{-1} .
$$

Kazhdan and Lusztig proved (in [7]) that for each $w \in W$ there exists a unique element $C_{w} \in H_{u}(W)$ such that $\overline{C_{w}}=C_{w}$ and

$$
C_{w}=\sum_{y \leq w}(-1)^{\ell(y)+\ell(w)} u^{\ell(w) / 2} u^{-\ell(y)} \overline{P_{y, w}} T_{y},
$$

where $P_{y, w} \in A$ is a polynomial on $u$ of degree $\leq \frac{1}{2}(\ell(w)-\ell(y)-1)$ for $y<w$, $P_{w, w}=1$, and " $\leq$ " is the Bruhat partial order on $W$. Furthermore, $\left\{C_{w} \mid w \in W\right\}$ forms an $A$-basis of $H_{u}(W)$ and is called the Kazhdan-Lusztig basis of $H_{u}(W)$, and the polynomial $P_{y, w}(u)$ is the well-known Kazhdan-Lusztig polynomial. For any field $F$ which is an $A$-algebra with $u^{1 / 2}$ specialized to $q^{1 / 2} \in F$, let $H_{q, F}(W)$ be the Iwahori-Hecke algebra associated to $W$ which is defined over $F$ and with Hecke parameter $q$. Then the elements in the set $\left\{T_{w} \otimes_{A} 1_{F} \mid w \in W\right\}$ (respectively, in the set $\left.\left\{C_{w} \otimes_{A} 1_{F} \mid w \in W\right\}\right)$ form an $F$-basis of $H_{q, F}(W)$. In the past decades 
these bases and polynomials have played important roles in many aspects of modern representation theory, cf. [6] and [7].

We now recall the definition of finite dihedral group.

Definition 3.1. Let $W_{n}:=I_{2}(n)$ be the finite dihedral group of order $2 n$, which is presented by the generators: $s, t$, and the following relations:

$$
s^{2}=t^{2}=1, \quad(s t)^{n}=1 .
$$

Let $H_{u}\left(W_{n}\right)$ be the corresponding Iwahori-Hecke algebra over $A$ with Hecke parameter $u$. As an $A$-algebra, $H_{u}\left(W_{n}\right)$ has a presentation with generators $T_{s}, T_{t}$ and the following relations:

$$
\left(T_{s}+1\right)\left(T_{s}-u\right)=0, \quad\left(T_{t}+1\right)\left(T_{t}-u\right)=0, \quad\left(T_{s} T_{t}\right)^{n}=\left(T_{t} T_{s}\right)^{n} .
$$

It is well-known that $H_{u}\left(W_{n}\right)$ is a free $A$-module with an $A$-basis $\left\{T_{w} \mid w \in I_{2}(n)\right\}$. For any field $F$ which is an $A$-algebra with $u^{1 / 2}$ specialized to $q^{1 / 2} \in F$, we shall often abbreviate $T_{w} \otimes_{A} 1_{F}$ and $C_{w} \otimes_{A} 1_{F}$ as $T_{w}$ and $C_{w}$ respectively.

The following result seems to be well-known to experts. For completeness, we add a proof here.

Lemma 3.2. Let $y, w \in W_{n}$. Then $P_{y, w}=1$ for all $y \leq w$. In particular,

$$
C_{w}=u^{-\ell(w) / 2} T_{w}+\sum_{w>y \in W_{n}}(-1)^{\ell(y)+\ell(w)} u^{\ell(w) / 2-\ell(y) / 2} u^{-\ell(y) / 2} T_{y} .
$$

Proof. We use induction on $\ell(w)$ to prove $P_{y, w}=1$. If $\ell(w)=0$, then $y \leq w$ implies that $y=w=1$ and hence $P_{y, w}=P_{w, w}=1$ in this case.

Suppose that $P_{y, w}=1$ holds for any $y \leq w$ and any $w \in W_{n}$ with $\ell(w)<k$. Now assume that $w \in W_{n}$ and $\ell(w)=k$. Let $y \leq w$. We want to show that $P_{y, w}=1$. Since $W_{n}=I_{2}(n)$ is generated by $\{s, t\}$. Without loss of generality, we can assume that $w=s v>v$. By $[7,2.2 . c]$,

$$
P_{y, w}=u^{1-c} P_{s y, v}+u^{c} P_{y, v}-\sum_{y \leq z \prec v, s z<z} \mu(z, v) u^{(\ell(v)-\ell(z)) / 2} u^{1 / 2} P_{y, z},
$$

where $c=1$ if $s y<y$; or $c=0$ if $s y>y$, and “ $\prec$ " is as defined in [7, Definition 1.2]. Note that $y \leq w=s v$ implies that either $y \leq v$ or $s y \leq v$. Therefore there are three possibilities:

Case 1. $y \not \$ v, s y \leq v$. Since $w=s v$ is of the form stst $\cdots$, it follows that this case happens if and only if $y=s v=w$. Thus $P_{y, w}=P_{w, w}=1$ as required.

Case 2. $y \leq v, s y \not \$ v$. Since $w=s v$ is of the form stst $\cdots$, it follows that this case happens if and only if $y=v$. Thus $P_{y, w}=P_{v, w}=P_{s w, w}=1$ by $[7$, Lemma $2.6($ iii)], as required. 
Case 3. $y \leq v, s y \leq v$. Since $w=s v$ is of the form stst $\cdots$, it follows that this case happens if and only if $y \leq t v<v$. By induction hypothesis, $P_{z, v}=1$ for any $z \leq v$, hence $z=t v$ is the unique element in $W_{n}$ such that $y \leq z \prec v, s z<z$ and $\mu(z, v) \neq 0$ and the definition of $\mu(z, v)$. In this case, $\mu(z, v)=1$. Hence

$$
P_{y, w}=1+u-u=1 .
$$

This completes the proof of the lemma.

Lemma 3.3. Let $w \in I_{2}(n)$. If $w \notin\{1, t\}$, then we have that

$$
T_{s} C_{w}= \begin{cases}-C_{w}, & \text { if } s w<w, \\ u C_{w}+u^{1 / 2} C_{s w}+u^{1 / 2} C_{t w}, & \text { if } s w>w .\end{cases}
$$

If $w=1$, then $T_{s} C_{1}=u C_{1}+u^{1 / 2} C_{s}$. If $w=t$, then $T_{s} C_{t}=u C_{t}+u^{1 / 2} C_{s t}$. The same is true if we interchange the role of $s$ and $t$.

Proof. By definition, $C_{s}=u^{-1 / 2} T_{s}-u^{1 / 2}$. It follows that $T_{s} C_{1}=u C_{1}+u^{1 / 2} C_{s}$. Similarly,

$$
C_{s t}=u^{-1} T_{s t}-T_{s}-T_{t}+u
$$

It follows that

$$
T_{s} C_{t}-u^{1 / 2} C_{s t}=T_{s}\left(u^{-1 / 2} T_{t}-u^{1 / 2}\right)-u^{1 / 2}\left(u^{-1} T_{s t}-T_{s}-T_{t}+u\right)=u C_{t},
$$

as required.

Now let $w \notin\{1, t\}$. Suppose that $s w>w$. By [7, (2.3.a)],

$$
T_{s} C_{w}=u C_{w}+u^{1 / 2} C_{s w}+u^{1 / 2} \sum_{\substack{z \prec w \\ s z<z}} \mu(z, w) C_{z},
$$

where $\mu(z, w)$ is defined to be the leading coefficient of the polynomial $P_{z, w}(u)$ and $\operatorname{deg} P_{z, w}=(\ell(w)-\ell(z)-1) / 2$.

Note that our assumption $w \notin\{1, t\}$ ensures that $\left\{z \in W_{n} \mid z \prec w, s z<z\right\} \neq \emptyset$. Let $z \in W_{n}$ such that $z \prec w$ and $s z<z$. In particular, $z \neq 1$. Since $W_{n}$ is the dihedral group with generating set $\{s, t\}$ and $P_{z, w}=1$ by Lemma 3.2, it follows (cf. [7, Lemma 2.6(iii)]) that our assumption forces that $\mu(z, w)=1, w=t z$ and hence $z=t w$ as required.

Let $i:=\sqrt{-1} \in \mathbb{C}$. Then $\zeta:=\exp (2 \pi i / n) \in \mathbb{C}$ is a primitive $n$-th root of unity in $\mathbb{C}$, and

$$
\zeta^{j}+\zeta^{-j}=2 \cos (2 j \pi / n) \in \mathbb{C}, \quad \forall j \in \mathbb{Z} .
$$

We set $K:=\mathbb{Q}[2 \cos (2 \pi / n)]\left(u^{1 / 2}\right)$. Let $\operatorname{Irr}\left(H_{u, K}\left(W_{n}\right)\right)$ be the set of isomorphism classes of irreducible $H_{u, K}\left(W_{n}\right)$-modules. 
Lemma 3.4. (see [3]) The Hecke algebra $H_{u, K}\left(W_{n}\right)$ is split semisimple. Furthermore, if $n$ is even, then $\operatorname{Irr}\left(H_{u, K}\left(W_{n}\right)\right)$ consists of the following four one-dimension representations and $(n-2) / 2$ two-dimensional representations:

$$
\begin{aligned}
& \rho_{0}^{(1)}: T_{s} \mapsto u, T_{t} \mapsto u, \quad \rho_{0}^{(2)}: T_{s} \mapsto-1, T_{t} \mapsto u, \\
& \rho_{0}^{(3)}: T_{s} \mapsto u, T_{t} \mapsto-1, \quad \rho_{0}^{(4)}: T_{s} \mapsto-1, T_{t} \mapsto-1, \\
& \rho_{j}: T_{s} \mapsto\left(\begin{array}{cc}
-1 & 0 \\
2 u+2 u \cos (2 j \pi / n) & u
\end{array}\right), T_{t} \mapsto\left(\begin{array}{cc}
u & 1 \\
0 & -1
\end{array}\right), 1 \leq j \leq \frac{n-2}{2} .
\end{aligned}
$$

If $n$ is odd, then $\operatorname{Irr}\left(H_{u, K}\left(W_{n}\right)\right)$ consists of the following two one-dimension representations and $(n-1) / 2$ two-dimensional representations:

$$
\begin{aligned}
& \rho_{0}^{(1)}: T_{s} \mapsto u, T_{t} \mapsto u, \quad \rho_{0}^{(2)}: T_{s} \mapsto-1, T_{t} \mapsto-1, \\
& \rho_{j}: T_{s} \mapsto\left(\begin{array}{cc}
-1 & 0 \\
2 u+2 u \cos (2 j \pi / n) & u
\end{array}\right), T_{t} \mapsto\left(\begin{array}{cc}
u & 1 \\
0 & -1
\end{array}\right), 1 \leq j \leq \frac{n-1}{2} .
\end{aligned}
$$

In [5], Graham and Lehrer introduced the notions of cellular bases and cellular algebras which capture the common feature of many important examples (including the Kazhdan-Lusztig basis and the Murphy basis for the type A Iwahori-Hecke algebras). A cellular structure on an algebra enables one to obtain a general description and systematic understanding of its irreducible representations and block theory by some unified linear algebra argument, which is very useful especially in the non-semisimple situation. It turns out that many important algebras in Lie theory fit in the framework of cellular algebras, see [5] and [9].

In the remaining part of this section, we shall use the main result of the last section to construct an explicit cellular basis of the Hecke algebras $H_{q, R}\left(W_{n}\right)$ associated to the dihedral group $W_{n}=I_{2}(n)$ over any commutative ring $R$ which is a $\mathbb{Z}\left[1 / n, 2 \cos (2 \pi / n), u^{ \pm 1 / 2}\right]$-algebra. Our bases will be some linear combinations of certain Kazhdan-Lusztig bases with coefficients given by evaluation of some explicit Chebyshev polynomials. Our construction is motivated by the work in [3], where Fakiolas gave a decomposition of the regular module of the semisimple Hecke algebra $H_{u, K}(W)$ over the field $\mathbb{Q}[\cos (\pi / n)]\left(u^{1 / 2}\right)$ into a direct sum of irreducible submodules. First, let's recall the definition of cellular algebras.

Definition 3.5. (see [5]) Let $R$ be a commutative domain and $A$ be an $R$-algebra which is free as an $R$-module. Let $(\Lambda, \succeq)$ be a finite poset. Suppose that for each $\lambda \in \Lambda$ there is a finite indexing set $T(\lambda)$, and for each pair $(\mathfrak{s}, \mathfrak{t})$ with $\mathfrak{s}, \mathfrak{t} \in T(\lambda)$ there is an element $c_{\mathfrak{s t}}^{\lambda} \in A$ such that the elements in the following set

$$
\left\{c_{\mathfrak{s t}}^{\lambda} \mid \lambda \in \Lambda \text { and } \mathfrak{s}, \mathfrak{t} \in T(\lambda)\right\}
$$


form an $R$-basis of $A$.

The basis $\left\{c_{\mathfrak{s t}}^{\lambda} \mid \lambda \in \Lambda\right.$ and $\left.\mathfrak{s}, \mathfrak{t} \in T(\lambda)\right\}$ is called a cellular basis of $A$ if

(C1) the $R$-linear map $*: A \mapsto A$ determined by $\left(c_{\mathfrak{s t}}^{\lambda}\right)^{*}=c_{\mathfrak{t s}}^{\lambda}$ for all $\lambda \in \Lambda$ and all $\mathfrak{s}, \mathfrak{t} \in T(\lambda)$, is an algebra anti-isomorphism of $A$; and

(C2) for any $\lambda \in \Lambda, \mathfrak{s}, \mathfrak{b} \in T(\lambda)$ and $a \in A$, there exists an element $r_{\mathfrak{b}} \in R$ such that for all $\mathfrak{t} \in T(\lambda)$

$$
a c_{\mathfrak{s t}}^{\lambda} \equiv \sum_{\mathfrak{b} \in T(\lambda)} r_{\mathfrak{b}} c_{\mathfrak{b t}}^{\lambda}\left(\bmod A^{\succ \lambda}\right)
$$

where $A^{\succ \lambda}$ denotes the $R$-submodule of $A$ spanned by the elements in the subset

$$
\left\{c_{\mathfrak{u} \mathfrak{v}}^{\mu} \mid \lambda \prec \mu \in \Lambda \text { and } \mathfrak{u}, \mathfrak{v} \in T(\mu)\right\} .
$$

If the $R$-algebra $A$ has a cellular basis over $R$ then $A$ is called a cellular algebra over $R$.

Assumption 3.6. Let $R$ be a commutative ring such that there is a ring homomorphism from $\theta: \mathbb{Z}\left[1 / n, 2 \cos (2 \pi / n), u^{ \pm 1 / 2}\right] \rightarrow R$. We set $q^{1 / 2}:=\theta\left(u^{1 / 2}\right)$, $p_{j}:=\theta(2 \cos (2 j \pi / n))$ for each $1 \leq j \leq[(n-1) / 2]$.

For any commutative ring $R$, we use $R^{\times}$to denote the set of invertible elements in $R$.

Corollary 3.7. With the Assumption 3.6 in mind, we have that $p_{l}-p_{j} \in R^{\times}$for any $1 \leq j<l \leq[(n-1) / 2]$. Furthermore, $2+p_{j} \in R^{\times}$for any $1 \leq j \leq[(n-1) / 2]$.

Proof. This follows directly from Lemmas 2.10 and 2.11 .

We are going to construct an explicit cellular basis for the Hecke algebra $H_{q, R}\left(W_{n}\right)$ over $R$ associated to $W_{n}=I_{2}(n)$. We shall consider the case when $n=2 m+1$ and the case when $n=2 m$ separately.

Case 1: Suppose that $n=2 m+1$. In this case, by our assumption, Lemma 2.10 and Corollary 3.7,

$$
\widetilde{S}_{m, R}(x)=\prod_{j=1}^{m}\left(x-p_{j}\right), \prod_{j=1}^{m}\left(2+p_{j}\right)=1,
$$

where $p_{1}, \cdots, p_{m}$ are pairwise distinct.

Definition 3.8. [3, Section 4] For any integers $1 \leq k \leq m-1$ and $1 \leq j \leq m$, we define

$$
a_{1}^{(j)}=S_{0, R}\left(p_{j}\right)=1, \quad a_{k+1}^{(j)}=\widetilde{S}_{k, R}\left(p_{j}\right)=S_{k, R}\left(p_{j}\right)+S_{k-1, R}\left(p_{j}\right) .
$$


By definition, we have that $a_{k}^{(j)} \in R$ for any $j, k$. Furthermore, applying (1) and the fact that $\widetilde{S}_{m, R}\left(p_{j}\right)=0$ we can get that $a_{m+1}^{(j)}=0$ and

$$
\begin{aligned}
a_{m}^{(j)} & =S_{m-1, R}\left(p_{j}\right)+S_{m-2, R}\left(p_{j}\right)=S_{m-1, R}\left(p_{j}\right)+p_{j} S_{m-1, R}\left(p_{j}\right)-S_{m, R}\left(p_{j}\right) \\
& =\left(2+p_{j}\right) S_{m-1, R}\left(p_{j}\right) .
\end{aligned}
$$

For each $2 \leq k \leq m-1$, by definition we have that

$$
a_{k}^{(j)}+a_{k+1}^{(j)}=S_{k-2, R}\left(p_{j}\right)+S_{k, R}\left(p_{j}\right)+2 S_{k-1, R}\left(p_{j}\right)=\left(p_{j}+2\right) S_{k-1, R}\left(p_{j}\right) .
$$

The same equality still holds when $k=1$.

Definition 3.9. (compare [3, Section 4]) For each $1 \leq j \leq m$, we define

$$
\begin{aligned}
& u_{j}:=\sum_{k=1}^{m} a_{k}^{(j)} C_{(s t)^{k-1} s} \in H_{q, R}\left(W_{n}\right), \\
& v_{j}:=\sum_{k=1}^{m-1}\left(a_{k}^{(j)}+a_{k+1}^{(j)}\right) C_{(t s)^{k}}+a_{m}^{(j)} C_{(t s)^{m}} \in H_{q, R}\left(W_{n}\right) .
\end{aligned}
$$

Lemma 3.10. With the notations as above, the elements in the set $\left\{u_{j}, v_{j} \mid 1 \leq\right.$ $j \leq m\}$ are $R$-linearly independent in $H_{q, R}\left(W_{n}\right)$ and form an $R$-basis of the space spanned by $\left\{C_{(s t)^{k-1} s}, C_{(t s)^{k}} \mid 1 \leq k \leq m\right\}$.

Proof. Let

$$
\begin{aligned}
X & :=\left(u_{1}, u_{2}, \cdots, u_{m}, v_{1}, v_{2}, \cdots, v_{m}\right)^{T}, \\
Y & :=\left(C_{s}, C_{s t s}, \cdots, C_{(s t)^{m-1} s}, C_{t s}, C_{(t s)^{2}}, \cdots, C_{(t s)^{m}}\right)^{T} .
\end{aligned}
$$

Then we can write $X=D Y$, where

$$
\begin{aligned}
& =\left(\begin{array}{ccccccccc}
a_{1}^{(1)} & a_{2}^{(1)} & \cdots & a_{m}^{(1)} & 0 & 0 & \cdots & 0 & 0 \\
a_{1}^{(2)} & a_{2}^{(2)} & \cdots & a_{m}^{(2)} & 0 & 0 & \cdots & 0 & 0 \\
\vdots & \vdots & \cdots & \vdots & \vdots & \vdots & \cdots & \vdots & \vdots \\
a_{1}^{(m)} & a_{2}^{(m)} & \cdots & a_{m}^{(m)} & 0 & 0 & \cdots & 0 & 0 \\
0 & 0 & \cdots & 0 & a_{1}^{(1)}+a_{2}^{(1)} & a_{2}^{(1)}+a_{3}^{(1)} & \cdots & a_{m-1}^{(1)}+a_{m}^{(1)} & a_{m}^{(1)} \\
0 & 0 & \cdots & 0 & a_{1}^{(2)}+a_{2}^{(2)} & a_{2}^{(2)}+a_{3}^{(2)} & \cdots & a_{m-1}^{(2)}+a_{m}^{(2)} & a_{m}^{(2)} \\
\vdots & \vdots & \vdots & \vdots & \vdots & \vdots & \cdots & \vdots & \vdots \\
0 & 0 & \cdots & 0 & a_{1}^{(m)}+a_{2}^{(m)} & a_{2}^{(m)}+a_{3}^{(m)} & \cdots & a_{m-1}^{(m)}+a_{m}^{(m)} & a_{m}^{(m)}
\end{array}\right) \\
& =\left(\begin{array}{cc}
A & 0 \\
0 & B
\end{array}\right),
\end{aligned}
$$


where

$$
\begin{aligned}
& A:=\left(\begin{array}{cccc}
a_{1}^{(1)} & a_{2}^{(1)} & \cdots & a_{m}^{(1)} \\
a_{1}^{(2)} & a_{2}^{(2)} & \cdots & a_{m}^{(2)} \\
\vdots & \vdots & \cdots & \vdots \\
a_{1}^{(m)} & a_{2}^{(m)} & \cdots & a_{m}^{(m)}
\end{array}\right), \\
& B:=\left(\begin{array}{ccccc}
a_{1}^{(1)}+a_{2}^{(1)} & a_{2}^{(1)}+a_{3}^{(1)} & \cdots & a_{m-1}^{(1)}+a_{m}^{(1)} & a_{m}^{(1)} \\
a_{1}^{(2)}+a_{2}^{(2)} & a_{2}^{(2)}+a_{3}^{(2)} & \cdots & a_{m-1}^{(2)}+a_{m}^{(2)} & a_{m}^{(2)} \\
\vdots & \vdots & \cdots & \vdots & \vdots \\
a_{1}^{(m)}+a_{2}^{(m)} & a_{2}^{(m)}+a_{3}^{(m)} & \cdots & a_{m-1}^{(m)}+a_{m}^{(m)} & a_{m}^{(m)}
\end{array}\right) .
\end{aligned}
$$

It is easy to see that $\operatorname{det} D=\operatorname{det} A \cdot \operatorname{det} B$ and $\operatorname{det} B=\operatorname{det} A$. Therefore, to prove the lemma, it suffices to show that $\operatorname{det}(B)$ is invertible in $R$.

By the discussion above Lemma 3.9, we have that for any $1 \leq k \leq m, a_{k}^{(j)}+$ $a_{k+1}^{(j)}=\left(p_{j}+2\right) S_{k-1, R}\left(p_{j}\right)$. Therefore, we can get that

$\operatorname{det} B$

$$
\begin{aligned}
& =\operatorname{det}\left(\begin{array}{ccccc}
p_{1}+2 & \left(p_{1}+2\right) p_{1} & \cdots & \left(p_{1}+2\right) S_{m-2, R}\left(p_{1}\right) & \left(p_{1}+2\right) S_{m-1, R}\left(p_{1}\right) \\
p_{2}+2 & \left(p_{2}+2\right) p_{2} & \cdots & \left(p_{2}+2\right) S_{m-2, R}\left(p_{2}\right) & \left(p_{2}+2\right) S_{m-1, R}\left(p_{2}\right) \\
\vdots & \vdots & \cdots & \vdots & \vdots \\
p_{m}+2 & \left(p_{m}+2\right) p_{m} & \cdots & \left(p_{m}+2\right) S_{m-2, R}\left(p_{m}\right) & \left(p_{m}+2\right) S_{m-1, R}\left(p_{m}\right)
\end{array}\right) \\
= & \left(\prod_{j=1}^{m}\left(p_{j}+2\right)\right) \operatorname{det}\left(\begin{array}{ccccc}
1 & p_{1} & \cdots & S_{m-2, R}\left(p_{1}\right) & S_{m-1, R}\left(p_{1}\right) \\
1 & p_{2} & \cdots & S_{m-2, R}\left(p_{2}\right) & S_{m-1, R}\left(p_{2}\right) \\
\vdots & \vdots & \cdots & \vdots & \vdots \\
1 & p_{m} & \cdots & S_{m-2, R}\left(p_{m}\right) & S_{m-1, R}\left(p_{m}\right)
\end{array}\right) .
\end{aligned}
$$

Since $S_{k}(x)=x^{k}+$ lower degree terms, it follows from an easy induction that

$$
\begin{aligned}
& \operatorname{det} B=\operatorname{det}\left(\begin{array}{ccccc}
1 & p_{1} & \cdots & S_{m-2, R}\left(p_{1}\right) & S_{m-1, R}\left(p_{1}\right) \\
1 & p_{2} & \cdots & S_{m-2, R}\left(p_{2}\right) & S_{m-1, R}\left(p_{2}\right) \\
\vdots & \vdots & \cdots & \vdots & \vdots \\
1 & p_{m} & \cdots & S_{m-2, R}\left(p_{m}\right) & S_{m-1, R}\left(p_{m}\right)
\end{array}\right)=\operatorname{det}\left(\begin{array}{cccc}
1 & p_{1} & \cdots & p_{1}^{m-1} \\
1 & p_{2} & \cdots & p_{2}^{m-1} \\
\vdots & \vdots & \cdots & \vdots \\
1 & p_{m} & \cdots & p_{m}^{m-1}
\end{array}\right) \\
= & \prod_{1 \leq i<j \leq m}^{m}\left(p_{j}-p_{i}\right) \in R^{\times},
\end{aligned}
$$

where the first step follows from Lemma 2.10. This completes the proof of the lemma. 
Definition 3.11. (compare [3, Section 4]) For each $1 \leq j \leq m$, we define

$$
\begin{aligned}
t_{j} & :=\sum_{k=1}^{m} a_{k}^{(j)} C_{(t s)^{k-1} t} \in H_{q, R}\left(W_{n}\right), \\
z_{j} & :=\sum_{k=1}^{m-1}\left(a_{k}^{(j)}+a_{k+1}^{(j)}\right) C_{(s t)^{k}}+a_{m}^{(j)} C_{(s t)^{m}} \in H_{q, R}\left(W_{n}\right) .
\end{aligned}
$$

Lemma 3.12. With the notations as above, the elements in the set $\left\{t_{j}, z_{j} \mid 1 \leq\right.$ $j \leq m\}$ are $R$-linearly independent in $H_{q, R}\left(W_{n}\right)$ and form an $R$-basis of the space spanned by $\left\{C_{(t s)^{k-1} t}, C_{(s t)^{k}} \mid 1 \leq k \leq m\right\}$.

Proof. This follows from a similar argument used in the proof of Lemma 3.10 by interchanging the role of $s$ and $t$.

Since $n=2 m+1$, by Lemma 3.4 the set $\operatorname{Irr}\left(H_{u, K}\left(W_{n}\right)\right)$ consists of two onedimensional representations and $m$ two-dimensional representations as follows:

$$
\begin{aligned}
& \rho_{0}^{(1)}: T_{s} \mapsto u, T_{t} \mapsto u, \\
& \rho_{0}^{(2)}: T_{s} \mapsto-1, T_{t} \mapsto-1, \\
& \rho_{j}: T_{s} \mapsto\left(\begin{array}{cc}
-1 & 0 \\
2 u+2 u \cos (2 j \pi / n) & u
\end{array}\right), T_{t} \mapsto\left(\begin{array}{cc}
u & 1 \\
0 & -1
\end{array}\right), \quad 1 \leq j \leq m .
\end{aligned}
$$

Set $\Lambda:=\{0, \infty, 1,2, \cdots, m\}$ which is in bijection $\iota$ with $\operatorname{Irr}\left(H_{u, K}\left(W_{n}\right)\right)$ via the following correspondence:

$$
0 \mapsto \rho_{0}^{(1)}, \quad \infty \mapsto \rho_{0}^{(2)}, \quad j \mapsto \rho_{j}, \forall 1 \leq j \leq m
$$

We define $0 \prec j \prec \infty$ for any $1 \leq j \leq m$. Thus we can define a partial order " $\preceq$ " on $\Lambda$ by

$$
\lambda \preceq \mu \Leftrightarrow \lambda=\mu \text { or } \lambda \prec \mu .
$$

Definition 3.13. Let $\lambda \in \Lambda:=\{0, \infty, 1,2, \cdots, m\}$ and set $T(\lambda)=\left\{1,2, \cdots, d_{\lambda}\right\}$, where $d_{\lambda}=\operatorname{dim}(\iota(\lambda))$. If $\lambda=0$, then $\iota(\lambda)=\rho_{0}^{(1)}$ and we define $m_{11}^{(0)}:=C_{1}=1$.

If $\lambda=\infty$, then $\iota(\lambda)=\rho_{0}^{(2)}$ and we define

$$
m_{11}^{(\infty)}:=C_{w_{0}}=C_{(s t)^{m} s}=C_{(t s)^{m} t} .
$$


If $\lambda=j$ for some $1 \leq j \leq m$, then $\iota(\lambda)=\rho_{j}$ is a two-dimensional representation. In this case, we define

$$
\begin{aligned}
& m_{11}^{(j)}:=u_{j}=\sum_{k=1}^{m} a_{k}^{(j)} C_{(s t)^{k-1} s}, \\
& m_{21}^{(j)}:=v_{j}=\left(\sum_{k=1}^{m-1}\left(a_{k}^{(j)}+a_{k+1}^{(j)}\right) C_{(t s)^{k}}+a_{m}^{(j)} C_{(t s)^{m}}\right), \\
& m_{12}^{(j)}:=z_{j}=\left(\sum_{k=1}^{m-1}\left(a_{k}^{(j)}+a_{k+1}^{(j)}\right) C_{(s t)^{k}}+a_{m}^{(j)} C_{(s t)^{m}}\right), \\
& m_{22}^{(j)}:=\left(2+p_{j}\right) t_{j}=\left(2+p_{j}\right) \sum_{k=1}^{m} a_{k}^{(j)} C_{(t s)^{k-1} t} .
\end{aligned}
$$

For each $\lambda \in \Lambda$, let $H_{q, R}^{\succ \lambda}$ and $H_{q, R}^{\succ \lambda}$ be the $R$-submodule of $H_{q, R}\left(W_{n}\right)$ generated by the elements in the set $\left\{m_{\mathfrak{u v}}^{(\mu)} \mid \mathfrak{u}, \mathfrak{v} \in T(\mu), \lambda \prec \mu \in \Lambda\right\}$ and the set $\left\{m_{\mathfrak{u v}}^{(\mu)} \mid \mathfrak{u}, \mathfrak{v} \in\right.$ $T(\mu), \lambda \preceq \mu \in \Lambda$ \} respectively. Recall that "*" is the anti-isomorphism of $H_{q, R}\left(W_{n}\right)$ which is defined on generators by $T_{w}^{*}=T_{w^{-1}}$ for all $w \in I_{2}(n)$.

Theorem 3.14. Suppose that $n=2 m+1$. We keep the Assumption 3.6 on $R$ and $n$ and the Definition 3.13. Then

1) for any $\lambda \in \Lambda, \mathfrak{s}, \mathfrak{t} \in T(\lambda)$, we have that $\left(m_{\mathfrak{s t}}^{(\lambda)}\right)^{*}=m_{\mathfrak{t s}}^{(\lambda)}$;

2) the elements in the set $\left\{m_{\mathfrak{s t}}^{(\lambda)} \mid \lambda \in \Lambda, \mathfrak{s}, \mathfrak{t} \in T(\lambda)\right\}$ are R-linearly independent and form an $R$-basis of $H_{q, R}\left(W_{n}\right)$;

$3)$ with the data of the anti-isomorphism “*”, the poset $(\Lambda, \succeq)$, and the set $T(\lambda)$ for each $\lambda \in \Lambda$, the set $\left\{m_{\mathfrak{s t}}^{(\lambda)}\right\}$ forms a cellular basis of $H_{q, R}\left(W_{n}\right)$.

Proof. 1) follows from the definition and a direct verification. Since

$$
\left\{C_{(s t)^{k-1} s}, C_{(t s)^{k}} \mid 1 \leq k \leq m\right\} \sqcup\left\{C_{(t s)^{k-1} t}, C_{(s t)^{k}} \mid 1 \leq k \leq m\right\} \sqcup\left\{C_{1}, C_{w_{0}}\right\}
$$

is a basis of $\left.H_{q, R}\left(W_{n}\right), 2\right)$ follows from Lemmas 3.10, 3.12, and Corollary 3.7.

It remains to prove 3 ). To this end, it suffices to verify the cellular axiom $\mathrm{C} 2$ ) in Definition 3.5.

Let $j \in \Lambda, \mathfrak{s} \in T(j)$. To verify the cellular axiom C2), it suffices to show that for each $\mathfrak{u} \in T(j)$, there exist $r_{\mathfrak{u}}, r_{\mathfrak{u}}^{\prime} \in R$, such that for any $\mathfrak{t} \in T(j)$,

$$
T_{s} m_{\mathfrak{s t}}^{(j)} \equiv \sum_{\mathfrak{u} \in T(j)} r_{\mathfrak{u}} m_{\mathfrak{u t}}^{(j)}\left(\bmod H_{q, R}^{\succ j}\right), \quad T_{t} m_{\mathfrak{s t}}^{(j)} \equiv \sum_{\mathfrak{u} \in T(j)} r_{\mathfrak{u}}^{\prime} m_{\mathfrak{u t}}^{(j)}\left(\bmod H_{q, R}^{\succ j}\right)
$$


If $j \in\{0, \infty\}$, then $T(j)=\{1\}$, and the above statement clearly holds. In fact, we have that

$$
\begin{aligned}
T_{s} m_{11}^{(0)} & =T_{s} C_{1}=q C_{1}+q^{1 / 2} C_{s} \equiv q m_{11}^{(0)}\left(\bmod H_{q, R}^{\succ 0}\right), \\
T_{t} m_{11}^{(0)} & =T_{t} C_{1}=q C_{1}+q^{1 / 2} C_{t} \equiv q m_{11}^{(0)}\left(\bmod H_{q, R}^{\succ 0}\right), \\
T_{s} m_{11}^{(\infty)} & =T_{s} C_{w_{0}}=-C_{w_{0}}=-m_{11}^{(\infty)} \\
T_{t} m_{11}^{(\infty)} & =T_{t} C_{w_{0}}=-C_{w_{0}}=-m_{11}^{(\infty)} .
\end{aligned}
$$

Henceforth, we assume that $j \in\{1,2, \cdots, m\}$.

By Lemma 3.3 we have that

$$
T_{s} m_{11}^{(j)}=T_{s} \sum_{k=1}^{m} a_{k}^{(j)} C_{(s t)^{k-1} s}=-\sum_{k=1}^{m} a_{k}^{j} C_{(s t)^{k-1} s}=-m_{11}^{(j)},
$$

and

$$
\begin{aligned}
& T_{t} m_{11}^{(j)} \\
= & T_{t} \sum_{k=1}^{m} a_{k}^{j} C_{(s t)^{k-1} s} \\
= & T_{t} C_{s}+T_{t} \sum_{k=2}^{m} a_{k}^{(j)} C_{(s t)^{k-1} s} \\
= & q C_{s}+q^{1 / 2} C_{t s}+\sum_{k=2}^{m} a_{k}^{(j)}\left(q C_{(s t)^{k-1} s}+q^{1 / 2} C_{(t s)^{k}}+q^{1 / 2} C_{(t s)^{k-1}}\right) \\
= & q m_{11}^{(j)}+q^{1 / 2}\left(\sum_{k=1}^{m-1}\left(a_{k}^{(j)}+a_{k+1}^{(j)}\right) C_{(t s)^{k}}+a_{m}^{(j)} C_{(t s)^{m}}\right) \\
= & q m_{11}^{(j)}+q^{1 / 2} m_{21}^{(j)}
\end{aligned}
$$

and

$$
\begin{aligned}
& T_{t} m_{21}^{(j)} \\
= & T_{t}\left(\sum_{k=1}^{m-1}\left(a_{k}^{(j)}+a_{k+1}^{(j)}\right) C_{(t s)^{k}}+a_{m}^{(j)} C_{(t s)^{m}}\right) \\
= & -\left(\sum_{k=1}^{m-1}\left(a_{k}^{(j)}+a_{k+1}^{(j)}\right) C_{(t s)^{k}}+a_{m}^{(j)} C_{(t s)^{m}}\right) \\
= & -m_{21}^{(j)}
\end{aligned}
$$

as required. 
Next, we want to compute $T_{s} m_{21}^{(j)}$. By definition, we have that

$$
\begin{aligned}
& T_{s} m_{21}^{(j)} \\
= & T_{s}\left(\sum_{k=1}^{m-1}\left(a_{k}^{(j)}+a_{k+1}^{(j)}\right) C_{(t s)^{k}}+a_{m}^{(j)} C_{(t s)^{m}}\right) \\
= & q\left(\sum_{k=1}^{m-1}\left(a_{k}^{(j)}+a_{k+1}^{(j)}\right) C_{(t s)^{k}}+\xi_{j}^{-1} a_{m}^{(j)} C_{(t s)^{m}}\right)+q^{1 / 2} a_{m}^{(j)} C_{w_{0}}+q^{1 / 2}\left(\left(a_{1}^{(j)}+a_{2}^{(j)}\right) C_{s}\right. \\
& \left.\quad+\sum_{k=1}^{m-2}\left(a_{k}^{(j)}+2 a_{k+1}^{(j)}+a_{k+2}^{(j)}\right) C_{(s t)^{k} s}+\left(a_{m-1}^{(j)}+2 a_{m}^{(j)}\right) C_{(s t)^{m-1} s}\right) \\
= & q m_{21}^{(j)}+q^{1 / 2} a_{m}^{(j)} C_{w_{0}}+q^{1 / 2}\left(\left(a_{1}^{(j)}+a_{2}^{(j)}\right) C_{s}+\sum_{k=1}^{m-2}\left(a_{k}^{(j)}+2 a_{k+1}^{(j)}+a_{k+2}^{(j)}\right) C_{(s t)^{k} s}\right. \\
& \left.+\left(a_{m-1}^{(j)}+2 a_{m}^{(j)}\right) C_{(s t)^{m-1} s}\right) \\
\equiv & q m_{21}^{(j)}+q^{1 / 2}\left(\left(a_{1}^{(j)}+a_{2}^{(j)}\right) C_{s}+\sum_{k=1}^{m-1}\left(a_{k}^{(j)}+2 a_{k+1}^{(j)}+a_{k+2}^{(j)}\right) C_{(s t)^{k} s}\right)\left(\bmod \left(H_{q, R}^{\succ j}\right)\right) .
\end{aligned}
$$

We claim that $a_{1}^{(j)}+a_{2}^{(j)}=\left(2+p_{j}\right) a_{1}^{(j)}$, and for each $1 \leq k \leq m-1$,

$$
a_{k}^{(j)}+2 a_{k+1}^{(j)}+a_{k+2}^{(j)}=\left(2+p_{j}\right) a_{k+1}^{(j)} .
$$

Once this is proved, we shall get that

$$
T_{s} m_{21}^{(j)} \equiv q m_{21}^{(j)}+q^{1 / 2}\left(2+p_{j}\right) m_{11}^{(j)}\left(\bmod \left(H_{q, R}^{\succ j}\right)\right),
$$

and we are done.

In fact, by Definition 3.8 and the paragraph above Definition 3.9, we have that $a_{1}^{(j)}+a_{2}^{(j)}=2+p_{j}=\left(2+p_{j}\right) a_{1}^{(j)}$. Furthermore, for each $1 \leq k \leq m-1$,

$$
\begin{aligned}
& a_{k}^{(j)}+2 a_{k+1}^{(j)}+a_{k+2}^{(j)}=\left(a_{k}^{(j)}+a_{k+1}^{(j)}\right)+\left(a_{k+1}^{(j)}+a_{k+2}^{(j)}\right) \\
= & \left(2+p_{j}\right) S_{k-1, R}\left(p_{j}\right)+\left(2+p_{j}\right) S_{k, R}\left(p_{j}\right)=\left(2+p_{j}\right)\left(S_{k-1, R}\left(p_{j}\right)+S_{k, R}\left(p_{j}\right)\right) \\
= & \left(2+p_{j}\right) a_{k+1}^{(j)},
\end{aligned}
$$

as required. As a consequence, we get (4). Thus we have that

$$
\begin{aligned}
& T_{s} m_{11}^{(j)}=-m_{11}^{(j)}, \quad T_{t} m_{21}^{(j)}=-m_{21}^{(j)}, \\
& T_{t} m_{11}^{(j)}=q m_{11}^{(j)}+q^{1 / 2} m_{21}^{(j)}, \\
& T_{s} m_{21}^{(j)} \equiv q m_{21}^{(j)}+q^{1 / 2}\left(2+p_{j}\right) m_{11}^{(j)}\left(\bmod \left(H_{q, R}^{\succ j}\right)\right) .
\end{aligned}
$$


Note that by definition $m_{12}^{(j)}, m_{22}^{(j)}$ can be obtained from $m_{21}^{(j)}, m_{11}^{(j)}$ by interchanging the role of $s, t$. By a symmetric argument, we can also get that

$$
\begin{aligned}
& T_{t} m_{22}^{(j)}=-m_{22}^{(j)}, \quad T_{s} m_{12}^{(j)}=-m_{12}^{(j)}, \\
& T_{s} m_{22}^{(j)}=q m_{22}^{(j)}+q^{1 / 2}\left(2+p_{j}\right) m_{12}^{(j)}, \\
& T_{t} m_{12}^{(j)} \equiv q m_{12}^{(j)}+q^{1 / 2} m_{22}^{(j)}\left(\bmod \left(H_{q, R}^{\succ j}\right)\right) .
\end{aligned}
$$

Comparing these equalities, we verified the Cellular Axiom (C2). Thus this completes the proof of the theorem.

Now we consider the construction of cellular basis of $H_{q, R}\left(W_{n}\right)$ in the case when $n=2 m$.

Case 2: Suppose that $n=2 m$. In this case, by our assumption, Lemma 2.11 and Corollary 3.7 ,

$$
S_{m-1, R}(x)=\prod_{j=1}^{m-1}\left(x-p_{j}\right), \quad \prod_{j=1}^{m-1}\left(2+p_{j}\right)=m,
$$

where $p_{1}, \cdots, p_{m-1}$ are pairwise distinct.

Definition 3.15. [3, Section 4] For any integers $1 \leq k \leq m-1$ and $1 \leq j \leq m-1$, we define

$$
\hat{a}_{1}^{(j)}=S_{0, R}\left(p_{j}\right)=1, \quad \hat{a}_{k}^{(j)}=S_{k-1, R}\left(p_{j}\right) .
$$

We also set $\hat{a}_{0}^{(j)}=\hat{a}_{m}^{(j)}:=0$.

Definition 3.16. (compare [3, Section 4]) For each $1 \leq j \leq m-1$, we define

$$
\begin{aligned}
u_{j}^{\prime} & =\sum_{k=0}^{m-1}\left(\hat{a}_{k}^{(j)}+\hat{a}_{k+1}^{(j)}\right) C_{(s t)^{k} s} \in H_{q, R}\left(W_{n}\right), \\
v_{j}^{\prime} & =\sum_{k=0}^{m-2}\left(\hat{a}_{k}^{(j)}+2 \hat{a}_{k+1}^{(j)}+\hat{a}_{k+2}^{(j)}\right) C_{(t s)^{k+1}} \in H_{q, R}\left(W_{n}\right), \\
t_{j}^{\prime} & =\sum_{k=0}^{m-1}\left(\hat{a}_{k}^{(j)}+\hat{a}_{k+1}^{(j)}\right) C_{(t s)^{k} t} \in H_{q, R}\left(W_{n}\right), \\
z_{j}^{\prime} & =\sum_{k=0}^{m-2}\left(\hat{a}_{k}^{(j)}+2 \hat{a}_{k+1}^{(j)}+\hat{a}_{k+2}^{(j)}\right) C_{(s t)^{k+1}} \in H_{q, R}\left(W_{n}\right) .
\end{aligned}
$$


Since $n=2 m$, by Lemma 3.4 the set $\operatorname{Irr}\left(H_{u, K}\left(W_{n}\right)\right)$ consists of four onedimensional representations and $m$ two-dimensional representations as follows:

$$
\begin{aligned}
& \rho_{0}^{(1)}: T_{s} \mapsto u, T_{t} \mapsto u, \quad \rho_{0}^{(2)}: T_{s} \mapsto-1, T_{t} \mapsto u, \\
& \rho_{0}^{(3)}: T_{s} \mapsto u, T_{t} \mapsto-1, \quad \rho_{0}^{(4)}: T_{s} \mapsto-1, T_{t} \mapsto-1, \\
& \rho_{j}: T_{s} \mapsto\left(\begin{array}{cc}
-1 & 0 \\
2 u+2 u \cos (2 j \pi / n) & u
\end{array}\right), T_{t} \mapsto\left(\begin{array}{cc}
u & 1 \\
0 & -1
\end{array}\right), \quad 1 \leq j \leq m-1 .
\end{aligned}
$$

Set $\Lambda:=\left\{0_{+}, 0_{-}, \infty_{+}, \infty_{-}, 1,2, \cdots, m-1\right\}$ which is in bijection $\iota$ with $\operatorname{Irr}\left(H_{u, K}\left(W_{n}\right)\right)$ via the following correspondence:

$$
\begin{aligned}
& 0_{-} \mapsto \rho_{0}^{(1)}, \quad 0_{+} \mapsto \rho_{0}^{(2)}, \quad \infty_{-} \mapsto \rho_{0}^{(3)}, \quad \infty_{+} \mapsto \rho_{0}^{(4)}, \\
& j \mapsto \rho_{j}, \quad \forall 1 \leq j \leq m .
\end{aligned}
$$

We define $0_{-} \prec 0_{+} \prec j \prec \infty_{-} \prec \infty_{+}$for any $1 \leq j \leq m-1$. Thus we can define a partial order " $\preceq$ " on $\Lambda$ by

$$
\lambda \preceq \mu \Leftrightarrow \lambda=\mu \text { or } \lambda \prec \mu .
$$

Definition 3.17. Let $\lambda \in \Lambda:=\left\{0_{+}, 0_{-}, \infty_{+}, \infty_{-}, 1,2, \cdots, m-1\right\}$ and set $T(\lambda)=$ $\left\{1,2, \cdots, d_{\lambda}\right\}$, where $d_{\lambda}=\operatorname{dim}(\iota(\lambda))$. If $\lambda=0_{-}$, then $\iota(\lambda)=\rho_{0}^{(1)}$ and we define $m_{11}^{\left(0_{-}\right)}:=C_{1}=1$.

If $\lambda=0_{+}$, then $\iota(\lambda)=\rho_{0}^{(2)}$ and we define

$$
m_{11}^{\left(0_{+}\right)}:=\sum_{k=1}^{m}(-1)^{k-1} C_{(s t)^{k-1} s} .
$$

If $\lambda=\infty_{-}$, then $\iota(\lambda)=\rho_{0}^{(3)}$ and we define

$$
m_{11}^{(\infty)}:=\sum_{k=1}^{m}(-1)^{k-1} C_{(t s)^{k-1} t}
$$

If $\lambda=\infty_{+}$, then $\iota(\lambda)=\rho_{0}^{(4)}$ and we define

$$
m_{11}^{(\infty)}:=C_{w_{0}}=C_{(s t)^{m}}=C_{(t s)^{m}} .
$$


If $\lambda=j$ for some $1 \leq j \leq m-1$, then $\iota(\lambda)=\rho_{j}$ is a two-dimensional representation. In this case, we define

$$
\begin{aligned}
& m_{11}^{(j)}:=u_{j}^{\prime}=\sum_{k=0}^{m-1}\left(\hat{a}_{k}^{(j)}+\hat{a}_{k+1}^{(j)}\right) C_{(s t)^{k} s} \\
& m_{21}^{(j)}:=v_{j}^{\prime}=\left(\sum_{k=0}^{m-2}\left(\hat{a}_{k}^{(j)}+2 \hat{a}_{k+1}^{(j)}+\hat{a}_{k+2}^{(j)}\right) C_{(t s)^{k+1}}\right) \\
& m_{22}^{(j)}:=\left(2+p_{j}\right) t_{j}^{\prime}=\left(2+p_{j}\right) \sum_{k=0}^{m-1}\left(\hat{a}_{k}^{(j)}+\hat{a}_{k+1}^{(j)}\right) C_{(t s)^{k} t}, \\
& m_{12}^{(j)}:=z_{j}^{\prime}=\left(\sum_{k=0}^{m-2}\left(\hat{a}_{k}^{(j)}+2 \hat{a}_{k+1}^{(j)}+\hat{a}_{k+2}^{(j)}\right) C_{(s t)^{k+1}}\right) .
\end{aligned}
$$

For each $\lambda \in \Lambda$, let $H_{q, R}^{\succ \lambda}$ and $H_{q, R}^{\succ \lambda}$ be the $R$-submodule of $H_{q, R}\left(W_{n}\right)$ generated by the elements in the set $\left\{m_{\mathfrak{u v}}^{(\mu)} \mid \mathfrak{u}, \mathfrak{v} \in T(\mu), \lambda \prec \mu \in \Lambda\right\}$ and the set $\left\{m_{\mathfrak{u v}}^{(\mu)} \mid \mathfrak{u}, \mathfrak{v} \in\right.$ $T(\mu), \lambda \preceq \mu \in \Lambda$ \} respectively. Recall that "*" is the anti-isomorphism of $H_{q, R}\left(W_{n}\right)$ which is defined on generators by $T_{w}^{*}=T_{w^{-1}}$ for all $w \in I_{2}(n)$.

We set $b_{0}^{(j)}:=0+\hat{a}_{1}^{(j)}=1$ and for any $1 \leq k \leq m-1$,

$$
b_{k}^{(j)}:=\hat{a}_{k}^{(j)}+\hat{a}_{k+1}^{(j)}=S_{k-1, R}\left(p_{j}\right)+S_{k, R}\left(p_{j}\right) .
$$

Lemma 3.18. With the notations as above, the elements in the set $\left\{m_{11}^{\left(0_{+}\right)}, m_{11}^{(j)} \mid 1 \leq\right.$ $j \leq m-1\}$ are $R$-linearly independent in $H_{q, R}\left(W_{n}\right)$ and form an $R$-basis of the space spanned by $\left\{C_{(s t)^{k}{ }_{s}} \mid 0 \leq k \leq m-1\right\}$.

Proof. By definition, we have that

$$
\left(m_{11}^{\left(0_{+}\right)}, m_{11}^{(1)}, m_{11}^{(2)}, \cdots, m_{11}^{(m-1)}\right)=\left(C_{s}, C_{s t s}, \cdots C_{\left.(s t)^{m-1} s\right)}\right) B
$$

where

$$
B:=\left(\begin{array}{ccccc}
1 & b_{0}^{(1)} & b_{0}^{(2)} & \cdots & b_{0}^{(m-1)} \\
-1 & b_{1}^{(1)} & b_{1}^{(2)} & \cdots & b_{1}^{(m-1)} \\
1 & b_{2}^{(1)} & b_{2}^{(2)} & \cdots & b_{2}^{(m-1)} \\
\vdots & \vdots & \vdots & \vdots & \vdots \\
(-1)^{m-1} & b_{m-1}^{(1)} & b_{m-1}^{(2)} & \cdots & b_{m-1}^{(m-1)}
\end{array}\right)
$$

It suffices to show that $\operatorname{det} B \in R^{\times}$. 
For $k=m-1, m-2, \cdots, 1$, we add the $k$ th row of $B$ to the $k+1$ th row of $B$ and thus get that

$$
\operatorname{det} B=\operatorname{det}\left(\begin{array}{ccccc}
1 & b_{0}^{(1)} & b_{0}^{(2)} & \cdots & b_{0}^{(m-1)} \\
0 & b_{1}^{(1)}+b_{0}^{(1)} & b_{1}^{(2)}+b_{0}^{(2)} & \cdots & b_{1}^{(m-1)}+b_{0}^{(m-1)} \\
0 & b_{2}^{(1)}+b_{1}^{(1)} & b_{2}^{(2)}+b_{1}^{(2)} & \cdots & b_{2}^{(m-1)}+b_{1}^{(m-1)} \\
\vdots & \vdots & \vdots & \vdots & \vdots \\
0 & b_{m-1}^{(1)}+b_{m-2}^{(1)} & b_{m-1}^{(2)}+b_{m-2}^{(2)} & \cdots & b_{m-1}^{(m-1)}+b_{m-2}^{(m-1)}
\end{array}\right) .
$$

By definition, $b_{0}^{(j)}+b_{1}^{(j)}=1+\hat{a}_{1}^{(j)}+\hat{a}_{2}^{(j)}=1+S_{0, R}\left(p_{j}\right)+S_{1, R}\left(p_{j}\right)=2+p_{j}$. Applying (1), $b_{k}^{(j)}+b_{k+1}^{(j)}=\left(2+p_{j}\right) S_{k, R}\left(p_{j}\right)$ for all $1 \leq k \leq m-2$. It follows that

$$
\begin{aligned}
\operatorname{det} B & =\operatorname{det}\left(\begin{array}{cccc}
2+p_{1} & 2+p_{2} & \cdots & 2+p_{m-1} \\
\left(2+p_{1}\right) S_{1, R}\left(p_{1}\right) & \left(2+p_{2}\right) S_{1, R}\left(p_{2}\right) & \cdots & \left(2+p_{m-1}\right) S_{1, R}\left(p_{m-1}\right) \\
\vdots & \vdots & \vdots & \vdots \\
\left(2+p_{1}\right) S_{m-2, R}\left(p_{1}\right) & \left(2+p_{2}\right) S_{m-2, R}\left(p_{2}\right) & \cdots & \left(2+p_{m-1}\right) S_{m-2, R}\left(p_{m-1}\right)
\end{array}\right) \\
& =\left(\prod_{j=1}^{m-1}\left(2+p_{j}\right)\right) \operatorname{det}\left(\begin{array}{cccc}
1 & 1 & \cdots & 1 \\
S_{1, R}\left(p_{1}\right) & S_{1, R}\left(p_{2}\right) & \cdots & S_{1, R}\left(p_{m-1}\right) \\
\vdots & \vdots & \vdots & \vdots \\
S_{m-2, R}\left(p_{1}\right) & S_{m-2, R}\left(p_{2}\right) & \cdots & S_{m-2, R}\left(p_{m-1}\right)
\end{array}\right) \\
& =m \operatorname{det}\left(\begin{array}{cccc}
1 & \cdots & 1 & \\
S_{1, R}\left(p_{1}\right) & S_{1, R}\left(p_{2}\right) & \cdots & S_{1, R}\left(p_{m-1}\right) \\
\vdots & \vdots & \vdots & \vdots \\
S_{m-2, R}\left(p_{1}\right) & S_{m-2, R}\left(p_{2}\right) & \cdots & S_{m-2, R}\left(p_{m-1}\right)
\end{array}\right) .
\end{aligned}
$$

On the other hand, by the definition of $S_{k, R}(x)$, we know that $S_{k, R}(x)=x^{k}+$ lower terms and $S_{1, R}(x)=x$. By an easy induction on $k$, we can deduce that

$$
\begin{array}{r}
\operatorname{det}\left(\begin{array}{cccc}
1 & 1 & \cdots & 1 \\
S_{1, R}\left(p_{1}\right) & S_{1, R}\left(p_{2}\right) & \cdots & S_{1, R}\left(p_{m-1}\right) \\
\vdots & \vdots & \vdots & \vdots \\
S_{m-2, R}\left(p_{1}\right) & S_{m-2, R}\left(p_{2}\right) & \cdots & S_{m-2, R}\left(p_{m-1}\right)
\end{array}\right) \\
=\operatorname{det}\left(\begin{array}{cccc}
1 & 1 & \cdots & 1 \\
p_{1} & p_{2} & \cdots & p_{m-1} \\
p_{1}^{2} & p_{2}^{2} & \cdots & p_{m-1}^{2} \\
\vdots & \vdots & \vdots & \vdots \\
p_{1}^{m-2} & p_{2}^{m-2} & \cdots & p_{m-1}^{m-2}
\end{array}\right)=\prod_{1 \leq i<j \leq m-1}\left(p_{j}-p_{i}\right) \in R^{\times},
\end{array}
$$

where the last inequality follows from Corollary 3.7. 
By Assumption 3.6, $m \in R^{\times}$. Therefore, we can conclude that $\operatorname{det} B \in R^{\times}$. This completes the proof of the lemma.

Lemma 3.19. The elements in the set $\left\{m_{12}^{(j)} \mid 1 \leq j \leq m-1\right\}$ are R-linearly independent in $H_{q, R}\left(W_{n}\right)$ and form an $R$-basis of the space spanned by $\left\{C_{(s t)^{k}} \mid 1 \leq\right.$ $k \leq m-1\}$.

Proof. It suffices to show that $\left\{z_{j}^{\prime} \mid 1 \leq j \leq m-1\right\}$ are $R$-linearly independent in $H_{q, R}\left(W_{n}\right)$ and form an $R$-basis of the space spanned by $\left\{C_{(s t)^{k}} \mid 1 \leq k \leq m-1\right\}$.

By definition, for each $1 \leq j \leq m-1$, we have that

$$
z_{j}^{\prime}=\sum_{k=0}^{m-2}\left(b_{k}^{(j)}+b_{k+1}^{(j)}\right) C_{(s t)^{k+1}}=\sum_{k=0}^{m-2}\left(2+p_{j}\right) S_{k}\left(p_{j}\right) C_{(s t)^{k+1}} .
$$

It follows that

$$
\left(z_{1}^{\prime}, z_{2}^{\prime}, \cdots, z_{m-1}^{\prime}\right)=\left(C_{s t}, C_{(s t)^{2}}, \cdots C_{\left.(s t)^{m-1}\right)}\right) P
$$

where

$$
P:=\left(\begin{array}{cccc}
2+p_{1} & \left(2+p_{1}\right) S_{1, R}\left(p_{1}\right) & \cdots & \left(2+p_{1}\right) S_{m-2, R}\left(p_{1}\right) \\
2+p_{2} & \left(2+p_{2}\right) S_{1, R}\left(p_{2}\right) & \cdots & \left(2+p_{2}\right) S_{m-2, R}\left(p_{2}\right) \\
\vdots & \vdots & \vdots & \vdots \\
2+p_{m-1} & \left(2+p_{m-1}\right) S_{1, R}\left(p_{m-1}\right) & \cdots & \left(2+p_{m-1}\right) S_{m-2, R}\left(p_{m-1}\right)
\end{array}\right) .
$$

It suffices to show that $\operatorname{det} P \in R^{\times}$. As in the proof of Lemma 3.19, we can deduce that

$$
\operatorname{det} P=\left(\prod_{j=1}^{m-1}\left(2+p_{j}\right)\right) \prod_{1 \leq i<j \leq m-1}\left(p_{j}-p_{i}\right)=m \prod_{1 \leq i<j \leq m-1}\left(p_{j}-p_{i}\right) \in R^{\times},
$$

as required. This completes the proof of the lemma.

Interchanging the role of $s, t$ in the proof of the above lemma we can get the following results.

Lemma 3.20. The elements in the set $\left\{m_{11}^{\left(\infty_{-}\right)}, m_{22}^{(j)} \mid 1 \leq j \leq m-1\right\}$ are $R$ linearly independent in $H_{q, R}\left(W_{n}\right)$ and form an $R$-basis of the space spanned by $\left\{C_{(t s)^{k} t} \mid 0 \leq k \leq m-1\right\}$.

Lemma 3.21. The elements in the set $\left\{m_{21}^{(j)} \mid 1 \leq j \leq m-1\right\}$ are R-linearly independent in $H_{q, R}\left(W_{n}\right)$ and form an $R$-basis of the space spanned by $\left\{C_{(t s)^{k}} \mid 1 \leq\right.$ $k \leq m-1\}$.

Theorem 3.22. Suppose that $n=2 m$. We keep the Assumption 3.6 on $R$ and $n$ and the Definition 3.17. Then 
1) for any $\lambda \in \Lambda, \mathfrak{s}, \mathfrak{t} \in T(\lambda)$, we have that $\left(m_{\mathfrak{s t}}^{(\lambda)}\right)^{*}=m_{\mathfrak{t s}}^{(\lambda)}$;

2) the elements in the set $\left\{m_{\mathfrak{s t}}^{(\lambda)} \mid \lambda \in \Lambda, \mathfrak{s}, \mathfrak{t} \in T(\lambda)\right\}$ are R-linearly independent and form an $R$-basis of $H_{q, R}\left(W_{n}\right)$;

3) with the data of the anti-isomorphism "*", the poset $(\Lambda, \succeq)$, and the set $T(\lambda)$ for each $\lambda \in \Lambda$, the set $\left\{m_{\mathfrak{s t}}^{(\lambda)}\right\}$ forms a cellular basis of $H_{q, R}\left(W_{n}\right)$.

Proof. 1) follows from the definition and a direct verification. Since

$$
\begin{aligned}
& \left\{C_{(s t)^{k} s} \mid 0 \leq k \leq m-1\right\} \sqcup\left\{C_{(t s)^{k} t} \mid 0 \leq k \leq m-1\right\} \sqcup\left\{C_{(s t)^{k}} \mid 1 \leq k \leq m-1\right\} \\
& \sqcup\left\{C_{(t s)^{k}} \mid 1 \leq k \leq m-1\right\} \sqcup\left\{C_{1}, C_{w_{0}}\right\}
\end{aligned}
$$

is a basis of $\left.H_{q, R}\left(W_{n}\right), 2\right)$ follows from Lemmas 3.18, 3.19, 3.20, and 3.21.

It remains to prove 3 ). To this end, it suffices to verify the cellular axiom C2) in Definition 3.5.

Let $j \in \Lambda, \mathfrak{s} \in T(j)$. To verify the cellular axiom C2), it suffices to show that for each $\mathfrak{u} \in T(j)$, there exist $r_{\mathfrak{u}}, r_{\mathfrak{u}}^{\prime} \in R$, such that for any $\mathfrak{t} \in T(j)$,

$$
T_{s} m_{\mathfrak{s t}}^{(j)} \equiv \sum_{\mathfrak{u} \in T(j)} r_{\mathfrak{u}} m_{\mathfrak{u t}}^{(j)}\left(\bmod H_{q, R}^{\succ j}\right), \quad T_{t} m_{\mathfrak{s t}}^{(j)} \equiv \sum_{\mathfrak{u} \in T(j)} r_{\mathfrak{u}}^{\prime} m_{\mathfrak{u t}}^{(j)}\left(\bmod H_{q, R}^{\succ j}\right) .
$$

If $j \in\left\{0_{ \pm}, \infty_{ \pm}\right\}$, then $T(j)=\{1\}$, and the above statement clearly holds. In fact, by Lemmas 3.18 and 3.20 , we have that

$$
\begin{aligned}
& T_{s} m_{11}^{\left(0_{-}\right)}=T_{s} C_{1}=q C_{1}+q^{1 / 2} C_{s} \equiv q m_{11}^{\left(0_{-}\right)}\left(\bmod H_{q, R}^{\succ 0_{-}}\right), \\
& T_{t} m_{11}^{\left(0_{-}\right)}=T_{t} C_{1}=q C_{1}+q^{1 / 2} C_{t} \equiv q m_{11}^{\left(0_{-}\right)}\left(\bmod H_{q, R}^{\succ 0_{-}}\right), \\
& T_{s} m_{11}^{\left(\infty_{+}\right)}=T_{s} C_{w_{0}}=-C_{w_{0}}=-m_{11}^{\left(\infty_{+}\right)}, \\
& T_{t} m_{11}^{\left(\infty_{+}\right)}=T_{t} C_{w_{0}}=-C_{w_{0}}=-m_{11}^{(\infty+)} .
\end{aligned}
$$

By Lemma 3.3, we have that

$$
T_{s} m_{11}^{\left(0_{+}\right)}=T_{s} \sum_{k=1}^{m}(-1)^{k-1} C_{(s t)^{k-1} s}=-m_{11}^{\left(0_{+}\right)},
$$

and

$$
\begin{aligned}
T_{t} m_{11}^{\left(0_{+}\right)} & =T_{t} \sum_{k=1}^{m}(-1)^{k-1} C_{(s t)^{k-1} s}=T_{t} C_{s}+T_{t} \sum_{k=2}^{m}(-1)^{k-1} C_{(s t)^{k-1} s} \\
& =q C_{s}+q^{1 / 2} C_{t s}+\sum_{k=2}^{m}(-1)^{k-1}\left(q C_{(s t)^{k-1} s}+q^{1 / 2} C_{(t s)^{k}}+q^{1 / 2} C_{(t s)^{k-1}}\right) \\
& =q m_{11}^{\left(0_{+}\right)}+(-1)^{m-1} q^{1 / 2} C_{w_{0}} \\
& \equiv q m_{11}^{\left(0_{+}\right)}\left(\bmod H_{q, R}^{\succ 0_{+}}\right) .
\end{aligned}
$$


Similarly, $T_{t} m_{11}^{\left(\infty_{-}\right)}=T_{s} \sum_{k=1}^{m}(-1)^{k-1} C_{(t s)^{k-1} t}=-m_{11}^{\left(\infty_{-}\right)}$, and

$$
\begin{aligned}
T_{s} m_{11}^{\left(\infty_{-}\right)} & =T_{s} \sum_{k=1}^{m}(-1)^{k-1} C_{(t s)^{k-1} t}=T_{s} C_{t}+T_{t} \sum_{k=2}^{m}(-1)^{k-1} C_{(t s)^{k-1} t} \\
& =q C_{t}+q^{1 / 2} C_{s t}+\sum_{k=2}^{m}(-1)^{k-1}\left(q C_{(t s)^{k-1} t}+q^{1 / 2} C_{(s t)^{k}}+q^{1 / 2} C_{(s t)^{k-1}}\right) \\
& =q m_{11}^{(\infty)}+(-1)^{m-1} q^{1 / 2} C_{w_{0}} \\
& \equiv q m_{11}^{\left(\infty_{-}\right)}\left(\bmod H_{q, R}^{\succ \infty}\right) .
\end{aligned}
$$

Henceforth, we assume that $j \in\{1,2, \cdots, m-1\}$. By Lemma 3.3, we have that

$$
T_{s} m_{11}^{(j)}=T_{s} \sum_{k=1}^{m-1} \hat{a}_{k}^{(j)}\left(C_{(s t)^{k-1} s}+C_{(s t)^{k} s}\right)=-m_{11}^{(j)},
$$

and

$$
\begin{aligned}
T_{t} m_{11}^{(j)} & =T_{t} \sum_{k=0}^{m-1}\left(\hat{a}_{k}^{(j)}+\hat{a}_{k+1}^{(j)}\right) C_{(s t)^{k} s}=T_{t} C_{s}+T_{t} \sum_{k=1}^{m-1}\left(\hat{a}_{k}^{(j)}+\hat{a}_{k+1}^{(j)}\right) C_{(s t)^{k} s} \\
& =q C_{s}+q^{1 / 2} C_{t s}+\sum_{k=1}^{m-1} b_{k}^{(j)}\left(q C_{(s t)^{k} s}+q^{1 / 2} C_{(t s)^{k+1}}+q^{1 / 2} C_{(t s)^{k}}\right) \\
& =q m_{11}^{(j)}+q^{1 / 2} \sum_{k=0}^{m-2}\left(b_{k}^{(j)}+b_{k+1}^{(j)}\right) C_{(t s)^{k+1}}+q^{1 / 2} \hat{a}_{m-1}^{(j)} C_{w_{0}} \\
& =q m_{11}^{(j)}+q^{1 / 2} m_{21}^{(j)}+q^{1 / 2} \hat{a}_{m-1}^{(j)} C_{w_{0}} \\
& \equiv q m_{11}^{(j)}+q^{1 / 2} m_{21}^{(j)}\left(\bmod H_{q, R}^{\succ j}\right),
\end{aligned}
$$

while $T_{t} m_{21}^{(j)}=T_{t} \sum_{k=0}^{m-2}\left(\hat{a}_{k}^{(j)}+2 \hat{a}_{k+1}^{(j)}+\hat{a}_{k+2}^{(j)}\right) C_{(t s)^{k+1}}=-m_{21}^{(j)}$, and

$$
\begin{aligned}
T_{s} m_{21}^{(j)} & =T_{s} \sum_{k=0}^{m-2}\left(\hat{a}_{k}^{(j)}+2 \hat{a}_{k+1}^{(j)}+\hat{a}_{k+2}^{(j)}\right) C_{(t s)^{k+1}} \\
& =q m_{21}^{(j)}+q^{1 / 2} \sum_{k=0}^{m-2}\left(\hat{a}_{k}^{(j)}+2 \hat{a}_{k+1}^{(j)}+\hat{a}_{k+2}^{(j)}\right)\left(C_{(s t)^{k+1} s}+C_{(s t)^{k} s}\right) \\
& =q m_{21}^{(j)}+q^{1 / 2} \sum_{k=0}^{m-2}\left(b_{k}^{(j)}+b_{k+1}^{(j)}\right)\left(C_{(s t)^{k+1} s}+C_{(s t)^{k} s}\right) \\
& =q m_{21}^{(j)}+q^{1 / 2} \sum_{k=0}^{m-2}\left(p_{j}+2\right) S_{k, R}\left(p_{j}\right)\left(C_{(s t)^{k+1} s}+C_{(s t)^{k} s}\right) \\
& =q m_{21}^{(j)}+q^{1 / 2}\left(p_{j}+2\right) \sum_{k=1}^{m-1} \hat{a}_{k}^{(j)}\left(C_{(s t)^{k} s}+C_{(s t)^{k-1} s}\right) \\
& =q m_{21}^{(j)}+q^{1 / 2}\left(p_{j}+2\right) m_{11}^{(j)} .
\end{aligned}
$$


Interchanging the role of $s$ and $t$, we can also get that

$$
\begin{aligned}
& T_{t} m_{22}^{(j)}=-m_{22}^{(j)}, \\
& T_{s} m_{22}^{(j)} \equiv q m_{22}^{(j)}+q^{1 / 2}\left(2+p_{j}\right) m_{12}^{(j)}\left(\bmod H_{q, R}^{\succ j}\right), \\
& T_{s} m_{12}^{(j)}=-m_{12}^{(j)}, \\
& T_{t} m_{12}^{(j)}=q m_{12}^{(j)}+q^{1 / 2} m_{22}^{(j)} .
\end{aligned}
$$

Comparing these equalities, we verified the Cellular Axiom (C2). Thus this completes the proof of the theorem.

Corollary 3.23. Suppose that $R=F$ is a field which satisfies the Assumption 3.6. Then the Hecke algebra $H_{q, F}\left(W_{n}\right)$ associated to the dihedral group $I_{2}(n)$ of order $2 n$ is split over $F$.

Proof. This follows from Theorems 3.14, 3.22, and general theory of cellular algebra $[5]$.

Acknowledgment. The authors would like to thank the referee for the valuable suggestions and comments.

\section{References}

[1] M. Abramowitz and I. A. Stegun, Handbook of Mathematical Functions, with Formulas, Graphs, and Mathematical Tables, Dover Publications, Inc., New York, 1966.

[2] G. Benkart and D. Moon, Tensor product representations of Temperley-Lieb algebras and Chebyshev polynomials, in: Representations of Algebras and Related Topics, in: Fields Inst. Commun., Amer. Math. Soc., Providence, RI, 45 (2005), 57-80.

[3] A. P. Fakiolas, The Lusztig isomorphism for Hecke algebras of dihedral type, J. Algebra, 126(2) (1989), 466-492.

[4] F. M. Goodman, P. de la Harpe and V. F. R. Jones, Coxeter Graphs and Towers of Algebras, Mathematical Sciences Research Institute Publications, 14, Springer-Verlag, New York, 1989.

[5] J. J. Graham and G. I. Lehrer, Cellular algebras, Invent. Math., 123(1) (1996), $1-34$.

[6] J. E. Humphreys, Reflection Groups and Coxeter Groups, Cambridge Studies in Advanced Mathematics, 29, Cambridge Univ. Press, Cambridge, UK, 1990.

[7] D. Kazhdan and G. Lusztig, Representations of Coxeter groups and Hecke algebras, Invent. Math., 53(2) (1979), 165-184. 
[8] J. C. Mason and D. C. Handscomb, Chebyshev Polynomials, Chapman \& Hall/CRC, Boca Raton, FL, 2003.

[9] E. Murphy, The representations of Hecke algebras of type $A_{n}$, J. Algebra, 173(1) (1995), 97-121.

Jun Hu (Corresponding Author) and Yabo Wu

School of Mathematics and Statistics

Beijing Institute of Technology

Beijing, 100081, P.R. China

emails: junhu303@qq.com (J. Hu)

15032910290@163.com (Y. Wu) 